Check for updates

Cite this: Phys. Chem. Chem. Phys. 2021, 23, 7926

Received 30th July 2020,

Accepted 21st December 2020

DOI: 10.1039/d0cp04051g

rsc.li/pccp

\title{
Morphological characterization and electronic properties of pristine and oxygen-exposed graphene nanoribbons on $\mathrm{Ag}(110)$
}

\author{
Jose Eduardo Barcelon, ${ }^{\text {ab }}$ Marco Smerieri, ${ }^{a}$ Giovanni Carraro, ${ }^{\text {ac }}$ \\ Pawel Wojciechowski, de Luca Vattuone, (D) ac Mario Rocca, *ac Silvia Nappini, \\ Igor Pišs, ${ }^{\text {fg }}$ Elena Magnano, (D) fh Federica Bondino, (D) Luca Vaghi, ${ }^{\mathrm{f}}$ Antonio Papagni \\ and Letizia Savio (iD *a
}

\begin{abstract}
Graphene nanoribbons (GNRs) are at the frontier of research on graphene materials since the 1D quantum confinement of electrons allows for the opening of an energy gap. GNRs of uniform and welldefined size and shape can be grown using the bottom-up approach, i.e. by surface assisted polymerization of aromatic hydrocarbons. Since the electronic properties of the nanostructures depend on their width and on their edge states, by careful choice of the precursor molecule it is possible to design GNRs with tailored properties. A key issue for their application in nanoelectronics is their stability under operative conditions. Here, we characterize pristine and oxygen-exposed $1.0 \mathrm{~nm}$ wide GNRs with a well-defined mixed edge-site sequence (two zig-zag and one armchair) synthesized on Ag(110) from 1,6-dibromo-pyrene precursors. The energy gap and the presence of quantum confined states are investigated by scanning tunneling spectroscopy. The effect of oxygen exposure under ultra-high vacuum conditions is inferred from scanning tunneling microscopy images and photoemission spectra. Our results demonstrate that oxygen exposure deeply affects the overall system by interacting both with the nanoribbons and with the substrate; this factor must be considered for supported GNRs under operative conditions.
\end{abstract}

\section{Introduction}

Graphene, a carbon allotrope consisting of a single layer of $\mathrm{sp}^{2}$ hybridized carbon atoms in a honeycomb lattice, shows relevant mechanical, electronic and optical properties (e.g. high intrinsic charge mobility, high electrical and thermal conductivity, high optical transparency and high tensile strength),

\footnotetext{
${ }^{a}$ IMEM-CNR, UOS Genova, Via Dodecaneso 33, 16146 Genova, Italy. E-mail: letizia.savio@imem.cnr.it,rocca@fisica.unige.it

${ }^{b}$ Dipartimento di Scienze Chimiche, della Vita e della Sostenibilità Ambientale Università di Parma, Parco Area delle Scienze, 17/A, 43124 Parma, Italy

${ }^{c}$ Dipartimento di Fisica, Università degli Studi di Genova, Via Dodecaneso 33, 16146 Genova, Italy

${ }^{d}$ Institute of Molecular Physics, Polish Academy of Sciences, M. Smoluchowskiego 17, 60-179 Poznań, Poland

${ }^{e}$ NanoBioMedical Centre, Adam Mickiewicz University, Wszechnicy Piastowskiej 3, 61-614 Poznań, Poland

${ }^{f}$ IOM CNR laboratorio TASC, 34149 Basovizza (TS), Italy

${ }^{g}$ Elettra-Sincrotrone Trieste S.C.p.A., 34149 Basovizza (TS), Italy

${ }^{h}$ Department of Physics, University of Johannesburg, PO Box 524 Auckland Park 2006, South Africa

${ }^{i}$ Dipartimento di Scienza dei Materiali, Università di Milano-Bicocca, Via R. Cozzi 55, 20125 Milano, Italy
}

which made it a promising material for several applications. However, while some of these properties (including excellent ballistic transport of charge carriers) make graphene suitable for devices where high electrical conductivity is mandatory (e.g. electrodes), the absence of a gap prevents its use in nanoelectronics, for which a semiconducting behaviour is required.

For this reason, the attention moved on low-dimensional graphene-based nanostructures such as carbon nanotubes and graphene nanoribbons (GNRs). The latter are graphene-like, one-dimensional (1D) nanostructures narrower than $10 \mathrm{~nm} .^{1-5}$ They are of particular interest because the quantum confinement of the electrons and the presence of different edge geometries (with possible associated electronic states) cause a bandgap opening, while still retaining the ballistic transport of charge carriers.

GNRs can be synthesized using several top-down methods, which include sonication, ${ }^{1}$ lithography ${ }^{6}$ and unzipping of carbon nanotubes (CNTs). ${ }^{7,8}$ These approaches allow for a relatively easy production of graphenic, low dimensional nanostructures which, however, often have a relatively poor quality in terms of uniformity and defectivity at the edges. Since regularity of shape and size is required for a variety of applications, and in particular for nanoelectronics, the alternative 
bottom-up approach appears more promising. ${ }^{9-12}$ It usually exploits the surface assisted polymerization of a molecular precursor (often a halogenated aromatic compound) catalyzed by a metal surface; the process occurs in two steps since Ullman-coupling is followed by cyclodehydrogenation and formation of new $\mathrm{C}-\mathrm{C}$ bonds. ${ }^{13-16}$

One of the first precursor molecules to be successfully employed was 10,10'-dibromo-9,9'-bianthracene (DBBA), which polymerizes forming $N=7$ GNRs with armchair edges. ${ }^{11}$ Eventually, a wide variety of halogenated and non-halogenated aromatic hydrocarbons ${ }^{17,18}$ has been employed to synthesize GNRs with well-defined width and edge sites, either armchair, zig-zag, ${ }^{19}$ mixed ${ }^{12}$ or cove. ${ }^{17}$ Chiral GNRs could also be produced starting from DBBA on $\mathrm{Cu}(111) .{ }^{10}$

The final product depends, indeed, on several factors, including the nature and geometry of the metallic substrate $\mathrm{e}^{11,20,21}$ and the structure of the precursor molecule. ${ }^{10-12,17,22-24}$ The choice of the proper compound is essential, since not all possible candidates are suitable and/or stable enough on the surface to allow for the dehydrogenation and $\mathrm{C}-\mathrm{C}$ coupling processes leading to the formation of extended graphenic nanostructures. As an example, we mention the case of 1,5 di-bromo-tetracene (DBT) and 1,6 di-bromo-pyrene (DBP), two halogenated aromatic hydrocarbons with similar molecular weight and different geometry: some of us produced ordered arrays of GNRs on $\mathrm{Ag}(110)$ from DBP polymerization, ${ }^{12}$ while desorption prevails for DBT on the same substrate. ${ }^{25}$ On the other hand, the use of $\mathrm{Br}$ corannulene on $\mathrm{Ag}(110)$ leads to the formation of C-based ribbons which do not have the spectroscopic signature of graphene because of the non-planar geometry of the precursor molecule. $^{24}$

It is therefore possible to engineer GNRs (and C-based nanostructures in general) of desired final geometry, and thus tailored electronic properties, by selecting suitable precursor molecules. Energy gaps may then range from close to zero up to almost $3 \mathrm{eV}^{19,23,26,27}$ depending on the width of the nanoribbons and on whether they present armchair (AGNRs) or zigzag (ZGNRs) edge sites. For AGNRs, the band gap depends on the ribbon width and the nanostructures can be classified in three groups yielding large, medium or small bandgap, respectively. ${ }^{23}$ On the contrary, ZGNRs have edge states associated with the zig-zag edge sites, ${ }^{19}$ for which a nearly flat dispersion and a magnetic behaviour are predicted. ${ }^{23}$ The determination of their electronic properties is therefore a pivotal task for the characterization of such nanostructures.

Another important issue is the chemical activity of GNRs. They are expected to be inert since their edges are saturated with hydrogen but, as in the case of graphene, their reactivity can be enhanced by doping with heteroatoms, ${ }^{16,28}$ thus opening perspectives in the fields of sensoristics and nano-catalysis.

Independently of their use, GNRs need to be stable under operative conditions. This implies, most often, after-growth exposure to air or a liquid solution and, possibly, their transfer from the native metal substrate to a more suitable substrate for the desired application. In this respect, we mention that GNRs can be successfully transferred with techniques similar to those employed for graphene films. ${ }^{29}$ For 7- and 9-AGNRs produced under ultra-high vacuum (UHV) conditions, the transfer process does not modify the morphology of the nanostructures. On the contrary, bottom-up synthesis of 7-AGNRs under less stringent, high vacuum (HV) conditions $\left(P=10^{-6} \mathrm{mbar}\right)^{30}$ is deeply affected by the presence of a residual partial pressure of water and oxygen. For these reasons, understanding at the fundamental level of the interaction of these nanostructures with atmospheric gases is a first step necessary to predict the structure and electronic properties of the nanoribbons under operative conditions and, if transfer from the native substrate is required, how these properties are affected by the substrate change.

Our manuscript deals with this field of research. In a previous Scanning Tunnelling Microscopy (STM) and Density Functional Theory (DFT) analysis, some of us demonstrated that mixed edge-sites GNRs synthesised on $\mathrm{Ag}(110)$ from DBP precursors have a very uniform width of $1.0 \mathrm{~nm}$ and a welldefined geometry alternating two zig-zag and one armchair site. ${ }^{12}$ Here, we complete their characterization by unravelling the semiconducting nature of the nanostructures through Scanning Tunnelling Spectroscopy (STS) measurements and we assess how the system is modified by exposure to oxygen in high vacuum conditions, as a first step towards understanding the behaviour of GNRs under realistic conditions. We measure a gap opening compatible with the one expected for graphene nanostructures of similar size and we report a double effect of oxygen exposure: it weakens the interaction of GNRs with the substrate by intercalation and formation of $\mathrm{C}=\mathrm{O}$ bonds and it also causes a partial modification of the $\mathrm{Ag}(110)$ surface morphology with the formation of $\mathrm{Ag}-\mathrm{O}$ complexes. The overall modification of the system must therefore be considered when exposing supported GNRs to reactive gases.

\section{Experimental}

Experiments were performed in three different UHV chambers equipped either for Low Temperature Scanning Tunneling Microscopy or for X-Ray Photoemission Spectroscopy (XPS).

The first setup consists of a preparation chamber, equipped with an evaporator for organic molecules, gas inlets, a quadrupole mass spectrometer and an ion gun for sample cleaning, and of a main chamber hosting the low temperature Scanning Tunneling Microscope (LT-STM manufactured by Createc) for STM and STS measurements.

The base pressure in the chamber is better than $1 \times 10^{-9}$ mbar but, since the LT-STM is totally shielded by a cold screen either at liquid nitrogen $\left(\mathrm{LN}_{2}, 77 \mathrm{~K}\right)$ or liquid helium temperature (LHe, $4 \mathrm{~K}$ ), the pressure at the scanner is much better and allows data acquisition on the same preparation for several days without significant sample contamination. STM images were recorded either at $\mathrm{LN}_{2}$ or LHe temperature, using a Pt/Ir tip cut under strain for the set of experiments performed to determine the surface morphology and a chemically etched tungsten (W) tip for STS measurements. In all cases the tip was 

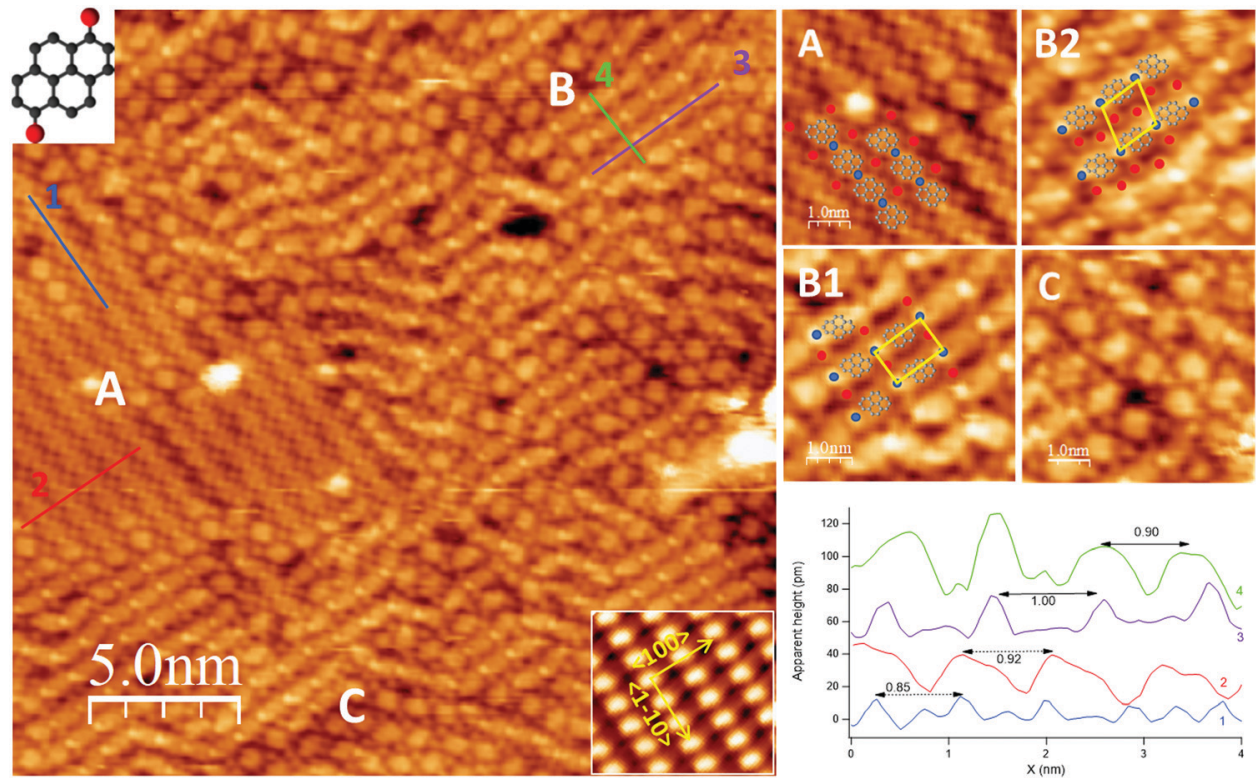

Fig. 1 Left: STM image ( $V=-150 \mathrm{meV}, I=0.3 \mathrm{nA})$ showing the co-existence of three different self-assembled structures (labelled as A, B, C) upon deposition of a sub-monolayer of DBP on $\mathrm{Ag}(110)$ at RT. Top and bottom insets report, respectively, the molecular structure of DBP and the atomically resolved image of clean $\mathrm{Ag}(110)$ from which the high symmetry directions (marked by arrows) are determined (image size: $1.8 \times 1.6 \mathrm{~nm}{ }^{2}$ ). Top right: enlarged image of each domain (either from the area in the overview or from neighbouring areas). Among B domains, it is possible to distinguish rectangular (B1) and rhomboidal unit cells (B2). Empirical models deduced from the periodicities of the self-assembled structures and from XPS results are superimposed to the STM image for structures A, B1 and B2 (grey: de-brominated pyrene units; blue: Ag adatoms; red: Br atoms). Bottom right: line scans cut across the paths marked in the overview for structures A and B1 (follow colour code). Line scans drawn along and across the rows of structure B2 are not reported since they do not differ significantly from those of B1. The higher and sharper features in traces 1 and 3 correspond to the Ag atoms within the organometallic chains.

repeatedly reshaped by controlled crashes into the surface, so that tunneling occurs effectively through an Ag-coated tip.

STM images were acquired in constant current mode, with a typical tunneling current of $0.1 \mathrm{nA}<I<1.0 \mathrm{nA}$ and a bias voltage $-1.5 \mathrm{~V}<V<1.5 \mathrm{~V}$ applied to the sample. No significant interaction between tip and sample was observed under these conditions, suggesting an overall stability of the system.

Surface orientation and image size were calibrated from atomically resolved images of the clean $\operatorname{Ag}(110)$ surface (see inset of Fig. 1A) or of the (nx1)-O-Ag(110) added row reconstruction. ${ }^{31,32}$ Similarly, heights were calibrated on monatomic Ag steps.

STS spectra and $\mathrm{d} I / \mathrm{d} V$ maps were recorded at LHe temperature to minimize thermal drift. The bias range $-0.5 \mathrm{~V}<V<$ $0.5 \mathrm{~V}$ was chosen to measure the gap of the GNRs and the relevant states within it with sufficient signal-to noise ratio and without appreciable drift of the sample. The range for $\mathrm{d} I / \mathrm{d} V$ maps was then extended to $-1.0 \mathrm{~V}<V<1.0 \mathrm{~V}$.

Data acquisition was performed using the STM-AFM software (ver. 3,1 provided by Createc). Images were processed using $\mathrm{WSxM}^{33}$ (version 9.4).

High-resolution synchrotron radiation XPS measurements on pristine nanostructures were performed at the BACH beamline of CNR at the Elettra synchrotron radiation source facility (Trieste, Italy). The samples were prepared and measured in an UHV chamber with a base pressure lower than $1 \times 10^{-9}$ mbar.
The XPS spectra were collected using a hemispherical electron energy analyser (VG Scienta, model R3000) at an angle of $60^{\circ}$ with respect to the X-ray incidence beam direction and at an emission angle of $60^{\circ}$ from the surface normal. $\mathrm{Br} 3 \mathrm{~d}$ and $\mathrm{C} 1 \mathrm{~s}$ core levels were acquired with 277 and $379 \mathrm{eV}$ photon energy, respectively. The binding energies are referenced to the substrate Fermi edge. The energy resolution of $0.15 \mathrm{eV}$ was determined from the width of the Fermi edge. The $\mathrm{C}$ 1s spectra were fitted with slightly asymmetric Doniach-Sunjic line shapes convoluted with the Gaussian profile. ${ }^{34,35}$ A Shirley type background was applied.

XPS spectra of the oxygen-exposed GNRs were acquired in a third UHV apparatus, equipped with a conventional, nonmonochromatized $\mathrm{Al}_{K \alpha}$ X-ray source and hemispherical analyzer (Omicron, DAR400 and EA125). Spectra were recorded at normal emission and calibrated to a binding energy of the $\mathrm{Ag}$ $3 \mathrm{~d}_{5 / 2}$ line of $368.25 \mathrm{eV}$.

\section{Sample preparation}

For the present experiments 1,6-Dibromopyrene (see inset in Fig. 1 for the molecular structure) was synthesized as described in ref. 12 .

The samples were prepared in situ in each experimental chamber. The Ag(110) surface was cleaned by cycles of sputtering with noble gas ions (either $\mathrm{Ne}^{+}$or $\mathrm{Ar}^{+}$with 1 to $3 \mathrm{keV}$ kinetic energy) followed by prolonged annealing to $T=550{ }^{\circ} \mathrm{C}$ or $T \sim 400{ }^{\circ} \mathrm{C}$ for microscopy and photoemission experiments, 
respectively. Surface cleanliness and order were checked by STM or by XPS and low energy electron diffraction.

DBP molecules were sublimated on the clean metal surface at room temperature (RT) from a Tantalum crucible heated to $70{ }^{\circ} \mathrm{C}-100{ }^{\circ} \mathrm{C}$ under UHV until a coverage of DBP on $\mathrm{Ag}(110)$ of $(0.6 \pm 0.2)$ ML was obtained, as estimated by XPS and STM. Sub-monolayer coverage is chosen in order to have some bare $\mathrm{Ag}(110)$ areas after GNRs polymerization, a condition preferable both for STS inspection and for better evidencing the effect of $\mathrm{O}_{2}$ exposure.

After DBP deposition, the sample was stepwise annealed to produce GNRs, following the protocol defined in ref. 12: firstly, the sample was slowly heated to $T=150{ }^{\circ} \mathrm{C}$ and kept at such temperature for $15 \mathrm{~min}$. Then, the temperature was raised to $400{ }^{\circ} \mathrm{C}\left(350{ }^{\circ} \mathrm{C}\right.$ for XPS experiments at $\left.\mathrm{BACH}\right)$ for $5 \mathrm{~min}$ and eventually slowly cooled down.

GNRs were subsequently exposed to $\mathrm{O}_{2}$ at RT or $300{ }^{\circ} \mathrm{C}$ for $20 \mathrm{~min}$ at a background pressure $P=1 \times 10^{-6} \mathrm{mbar}$, resulting in a nominal exposure of $900 \mathrm{~L}$. Since a doser placed at $\sim 2 \mathrm{~cm}$ from the sample surface was employed, we estimate a local enhancement of the pressure by approximately a factor of $5 .^{36}$

At each stage of the preparation the sample was cooled down to $\mathrm{LN}_{2}$ temperature and inserted eventually into the LT-STM for inspection. Conversely, XPS spectra were recorded with the sample at RT.

\section{Results and discussion}

\section{(i) $\mathrm{DBP} / \mathrm{Ag}(110)$ at $\mathrm{RT}$}

Upon deposition of a sub-monolayer of DBP at RT, the precursor molecules self-assemble into large islands, often covering the whole terrace, and organize in domains with different geometries. Such behavior denotes a large mobility of the precursor molecules at RT.

The overview in Fig. 1 shows the co-existence of three different self-assembled structures (denoted as A, B, C in the image), which are better detailed in the enlargements on the right-hand side of the figure. Among B domains, it is possible to distinguish rectangular (B1) and rhomboidal unit cells (B2), shown in the top right panel. Structures A, B1 and B2 form ordered patterns with specific periodicities, as shown in the line scans in the bottom panel. Structure C presents a less regular assembly; it appears less frequently and it is found in small domains at the border between domains of type A and B.

Structure A consists of straight rows oriented, within experimental error, along the $\langle\mathbf{1} \overline{1} 0\rangle$ direction. Nearly oval features alternate to small, bright dots; statistical analysis over several line scans, as the one reported in the bottom panel, yields a periodicity of $(8.4 \pm 0.9) \AA$ (trace 1 , blue) and $(9.0 \pm 0.4) \AA$ (trace 2, red) along and across the rows, respectively, from which we infer a local molecular density of $\sim 1.3 \times 10^{14}$ DBP units per $\mathrm{cm}^{2}$. These periodicities suggest that each of the large roundish protrusions in the chain consists of one single DBP unit. Additional bright small features are also present in-between the rows, the nature of which is discussed in the following.

Structure B consists of chains alternating a larger protrusion, identified with a DBP unit, with a smaller and brighter one. One or two additional spots per each DBP unit are present in-between the rows. At variance with structure A, these chains are oriented at either $-35^{\circ}$ or $88^{\circ}$ off $\langle 1 \overline{1} 0\rangle$ and they are rather bent than straight. The less regular geometry causes the DBP units in adjacent rows to be either aligned, giving rise to a rectangular unit cell (assembly B1), or misaligned, resulting in a rhomboidal unit cell (assembly B2). Statistical analysis over high-resolution images of structure $\mathrm{B}$ allowed to determine an average periodicity of $(9.9 \pm 0.5) \AA$ along the rows (trace 3, violet) and $(8.7 \pm 0.5) \AA$ across the rows (trace 4 , green), corresponding to a local molecular density of $\sim 1.2 \times 10^{14}$ DBP units per $\mathrm{cm}^{2}$.

XPS was used to determine the possible chemical nature of the self-assembled DBP layer. Upon deposition of $0.6 \mathrm{ML}$ of DBP on $\mathrm{Ag}(110)$ at RT (bottom spectra in Fig. 2a and b), the $\mathrm{Br}$ $3 \mathrm{~d}$ spectral region shows the presence of a major $\mathrm{Br}$ moiety evidenced by the $3 \mathrm{~d}$ doublet with $E_{\mathrm{b}}\left(\mathrm{Br} 3 \mathrm{~d}_{5 / 2}\right)=68.2 \mathrm{eV}$ and a minority one with $E_{\mathrm{b}}\left(\mathrm{Br} 3 \mathrm{~d}_{5 / 2}\right)=70.4 \mathrm{eV}$. The latter component is ascribed to $\mathrm{Br}-\mathrm{C}$ bonds in the intact $\mathrm{DBP}$ molecules, as substantiated by comparison with the corresponding experiment performed at $1.2 \mathrm{ML}$ DBP coverage $^{12}$ and by other experiments performed on the less reactive $\mathrm{Au}(111)$ surface. $^{37,38}$ The lower $E_{\mathrm{b}}$ species corresponds, on the other hand, to $\mathrm{Br}$ atoms chemisorbed on the $\mathrm{Ag}$ substrate and its higher intensity indicates an almost complete debromination of the DBP admolecules at RT. This picture is confirmed by

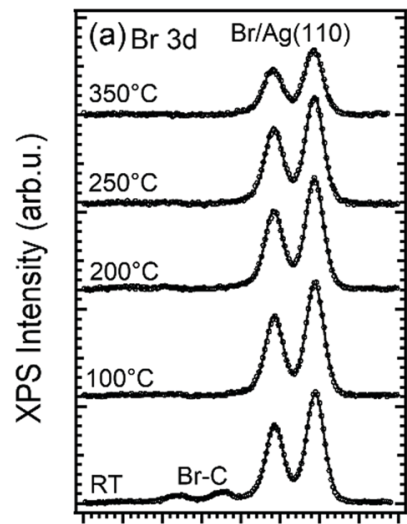

747372717069686766 Binding energy (eV)

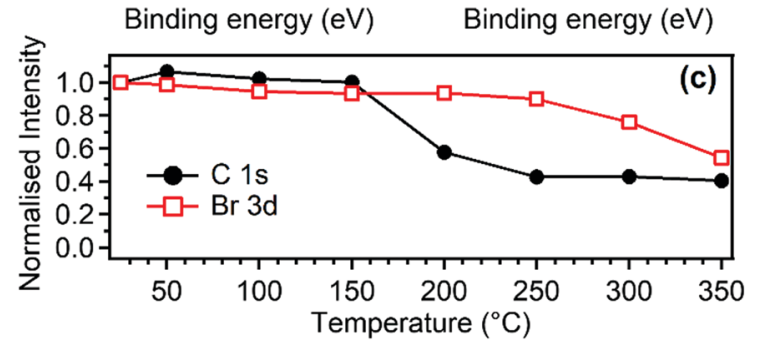

Fig. 2 (a) $B r 3 d$ and (b) $C$ 1s core level spectra of $0.6 \mathrm{ML}$ of DBP deposited on $\mathrm{Ag}(110)$ at $\mathrm{RT}$ and after annealing the sample to the indicated temperature for $5 \mathrm{~min}$. (c) Normalized integrated intensity of $\mathrm{Br} 3 \mathrm{~d}$ and $C$ 1s peaks as a function of temperature. The intensities are normalized to the peak areas measured after the deposition. 
inspection of the $\mathrm{C} 1 \mathrm{~s}$ spectrum. The main peak at $E_{\mathrm{b}} \sim 284.3 \mathrm{eV}$ corresponds to the aromatic carbon rings. In addition, a weaker but clearly visible component is present at $283.1 \mathrm{eV}$, which is a typical fingerprint of $\mathrm{C}-\mathrm{Ag}$ bonds that are formed when metal adatoms from the substrate react with carbon radicals. This is, therefore, a signature that breaking of the $\mathrm{C}-\mathrm{Br}$ bond has occurred ${ }^{25,39}$ and that DBP-Ag organometallic chains have formed. Peak decomposition analysis reveals that the main peak results from the superposition of at least three unresolved components. The relative intensities indicate that the two components at $\sim 284.1$ and $\sim 284.5 \mathrm{eV}$ could be assigned to carbon atoms bound only to carbon and to carbon atoms bound to carbon and hydrogen, respectively. The third component at $E_{\mathrm{b}} \sim 285.2 \mathrm{eV}$ is rather weak and we ascribe it to $\mathrm{Br}-\mathrm{C}$ bonds. In accordance with $\mathrm{Br} 3 \mathrm{~d}$ spectra, it disappears after a short mild annealing or after some hours at room temperature (not shown). In this respect we must note that the relative integrated intensity of the $\mathrm{C}-\mathrm{Ag}$ component with respect to the total $\mathrm{C}$ 1s peak area (0.06) is about half of the value expected for complete substitution of all the $\mathrm{C}-\mathrm{Br}$ bonds with $\mathrm{C}-\mathrm{Ag}$ bonds (0.125). Thus, XPS data suggest that at submonolayer coverage and RT the large majority of the DBP molecules undergo complete debromination and that the detached $\mathrm{Br}$ atoms are adsorbed on the substrate, but that not all the broken $\mathrm{C}$ bonds are saturated by Ag. This can be explained by the formation of surface stabilized pyrene radicals or biradicals adsorbed in conformations not favorable for bonding with the $\mathrm{Ag}$ adatoms. Formation of covalent bonds between the pyrene derivatives is less probable in view of the morphology observed by STM and of the models proposed in the following.

The XPS information is valuable for a complete interpretation of the STM image of Fig. 1, since it allows to identify the small bright features present in and in-between the rows. Therefore, by analogy with what observed for a DBP multilayer annealed to $150{ }^{\circ} \mathrm{C},{ }^{12}$ we propose that the de-brominated molecules form organometallic chains with Ag adatoms provided by the sample, most probably released from the steps, ${ }^{32}$ while dissociated $\mathrm{Br}$ atoms sit in-between these chains. So, the empirical models superimposed to the enlargements in Fig. 1 are proposed: the bright dots in the chains are $\mathrm{Ag}$ atoms (blue circles) bound to the pyrene groups to form organometallic proto-polymers and the $\mathrm{Br}$ atoms occupy interstitial sites inbetween the rows (red circles).

\section{(ii) Synthesis and characterization of GNRs}

The preparation reported in Fig. 1 was eventually annealed following the protocol described in ref. 12 and summarized in the experimental section, in order to induce the synthesis of GNRs by surface assisted polymerization (Fig. 3a). The so-produced sample was then exposed to oxygen at RT and at $T=300{ }^{\circ} \mathrm{C}$ (Fig. $3 \mathrm{~b}$ and c, respectively), as discussed in the next section.

For the pristine GNRs covered sample (panel a), we firstly mention that the nanostructures obtained starting from a submonolayer coverage of the precursor molecules have the same chemical nature as those observed starting from a DBP multilayer, ${ }^{12}$ as witnessed by the evolution of the XPS spectra upon annealing the DBP layer. Indeed, as evident from Fig. 2, debromination is fully completed after mild annealing to $100{ }^{\circ} \mathrm{C}$. The organometallic phase is rather stable up to $200{ }^{\circ} \mathrm{C}$, although the central $\mathrm{C} 1 \mathrm{~s}$ peak is changing and the fitting with two components as described above slightly deviates from the experimental data. These intermediate steps could be related to the transformation between the different structures observed by STM. More research is necessary to understand these transition states, which goes beyond the scope of the current work. For $200{ }^{\circ} \mathrm{C}<T<250{ }^{\circ} \mathrm{C}$, we observe the disappearance of the $\mathrm{C}-\mathrm{Ag}$ component in the $\mathrm{C} 1 \mathrm{~s}$ spectrum while the main peak shifts towards higher binding energy, mainly due to the change in the surface work function and energy level alignment at the adsorbate/substrate interface. ${ }^{12}$ This is indicative of a chemical transformation of the deposit, which is correlated with the change in surface morphology observed by STM and identified with the beginning of the polymerization process. ${ }^{12}$ Since, at the same time, the C $1 \mathrm{~s}$ intensity drops significantly [Fig. 2(c)], polymerization must compete with thermal desorption of the precursor molecules. Annealing to $350{ }^{\circ} \mathrm{C}$ does not further reduce the amount of $\mathrm{C}$ containing species, indicating a higher stability of the bonding
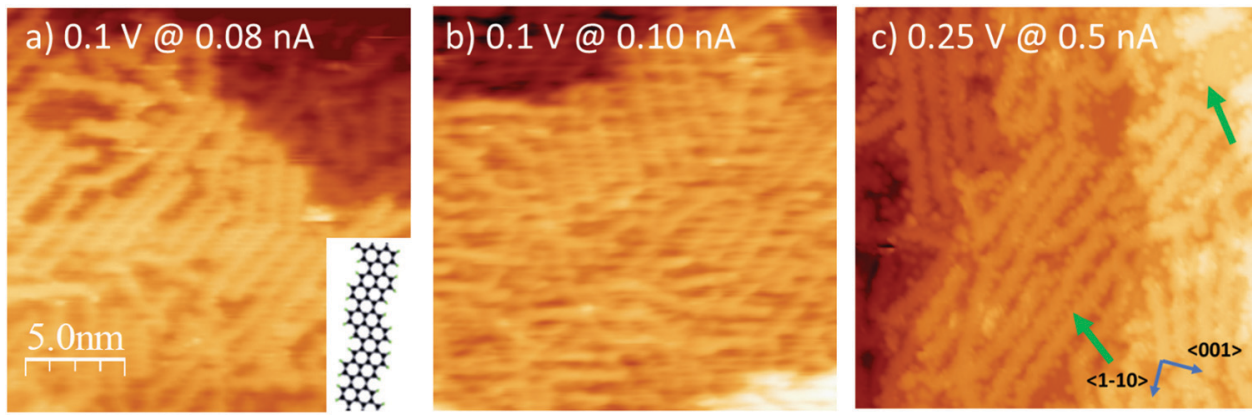

Fig. 3 STM images showing overviews of the $\mathrm{Ag}(110)$ surface covered by a sub-monolayer of GNRs and subject to different treatments: (a) pristine GNRs; (b) same as (a) after exposure to $\mathrm{O}_{2}$ at room temperature; (c) same as (a) after exposure to $\mathrm{O}_{2}$ at $300{ }^{\circ} \mathrm{C}$. The nominal $\mathrm{O}_{2}$ dose is always $900 \mathrm{~L}$ ( $\sim 500 \mathrm{~L}$ when considering the doser enhancement factor). The green arrows in (c) point to additional features forming both in-between the GNRs and on bare $\mathrm{Ag}(110)$ areas. 
of the structure with the substrate when compared with the molecular and organometallic precursors present at lower $T$.

At high temperature the $\mathrm{C}$ 1s spectrum broadens. Such effect can be assigned to the increasing amount of inequivalent $\mathrm{C}$ atoms with respect to the substrate but also to a partial decomposition of the reactants. Finally, we mention that the amount of bromine on the surface reduces by $\sim 50 \%$ upon annealing to $350{ }^{\circ} \mathrm{C}$ and it is expected to disappear completely at even higher temperatures since $\mathrm{Br}$ is known to recombine with $\mathrm{H}$ atoms and desorb as soon as $\mathrm{C}$ dehydrogenation takes place and polymerization sets in. ${ }^{12,40}$

STM inspection reveals that the nanoribbons arrange at the surface in arrays oriented parallel to each other to form locally ordered patterns. The estimated reduction with respect to the initial DBP coverage is apparently smaller in STM images than deduced from XPS inspection, but this is probably related to the slightly different heating rate in the two experiments or to the uncertainty $(0.6 \pm 0.2 \mathrm{ML})$ in the initial DBP coverage. The overall degree of order on the surface is lower than for the case in which GNRs were produced starting from a DBP multilayer; ${ }^{12}$ the less packed arrangement of the GNRs, however, may facilitate intercalation of $\mathrm{O}_{2}$. In addition, we remind that having some bare $\mathrm{Ag}(110)$ areas is preferable when performing STS measurements.

GNRs grown by surface assisted polymerization of DBP precursors are very narrow $(\sim 1.0 \mathrm{~nm})$ and have a peculiar edge-site sequence, which alternates two zigzag and one armchair site. ${ }^{12}$ Since the width of the GNRs and the presence of edge-states are known to determine the size of the bandgap, we performed local STS measurements and $\mathrm{d} I / \mathrm{d} V$ maps of the GNRs to retrieve this information. The STM image in Fig. 4a shows the morphology of the GNRs. The crosses indicate on which nanostructure and at which point the STS spectra were recorded. Such spectra are reported in panel b and show the presence of a gap of width $\Delta V \sim 530 \mathrm{meV}$ approximately centred around the Fermi energy, $E_{\mathrm{F}}$. The opening of a gap is expected because of the $1 \mathrm{D}$ confinement of electronic states in the GNRs. We note that the gap is slightly asymmetric with respect to the Fermi edge towards positive $\mathrm{V}$, corresponding to tunnelling into empty states of the sample. This is not sufficient to determine if the GNRs are undoped or weakly n-doped; however, n-doping is expected for graphene on $\mathrm{Ag}^{41}$ and the binding energy of the $\mathrm{C} 1 \mathrm{~s}$ peak upon polymerization is consistent with n-doped graphene structures. ${ }^{42,43}$ Moreover, additional weak states are present within the gap at $-50 \mathrm{mV}$ and $41 \mathrm{meV}$ and at the negative edge of the gap at $\sim-250 \mathrm{mV}$. We ascribe these features to GNRs. A contribution from the STM tip can be ruled out since the corresponding $\mathrm{d} I / \mathrm{d} V$ curve recorded on clean $\mathrm{Ag}(110)$ is completely flat, as expected by comparison with literature data. ${ }^{44}$ Based on the STS spectra of panel (b), $\mathrm{d} I / \mathrm{d} V$ maps were recorded at bias voltage approximately corresponding to these features and to the edges of the energy gap (see Fig. 4c). These $\mathrm{d} I / \mathrm{d} V$ maps show changes in the image contrast at different V. For negative bias, which allows to probe occupied states (i.e. the HOMO molecular orbitals), there is a distinct corrugation of the $\mathrm{d} I / \mathrm{d} V$ map of the nanoribbon. At $V=-50 \mathrm{meV}$, the image appears more contrasted and some intense bright spots appear along the nanoribbons; they are especially evident in the short GNR on which STS spectra were recorded, where they repeat three times with an almost regular spacing. Such behaviour indicates that locally there is a larger contribution of that state to the HOMO of the GNR. At $-250 \mathrm{meV}$, the same features appear, although with attenuated brightness. Therefore, the states generating the modulation of the LDOS when hopping across GNR are different, but they are spatially localized in the same portion of nanoribbon. For the unoccupied states (LUMO), i.e. for $\mathrm{d} I / \mathrm{d} V$ maps recorded at 50 and $260 \mathrm{mV}$, the contrast is lower and more uniform.

The measured energy gap is relatively small but it falls well in the range expected for GNRs. ${ }^{16,23}$ We mention that, though some trends have been determined for the relation between the gap opening and the GNR width, they refer essentially to AGNRs, while much less information is available for nanostructures with different edges.

Identifying the nature of the states within the gap, especially the one at $-50 \mathrm{meV}$, on the contrary, is not straightforward. On
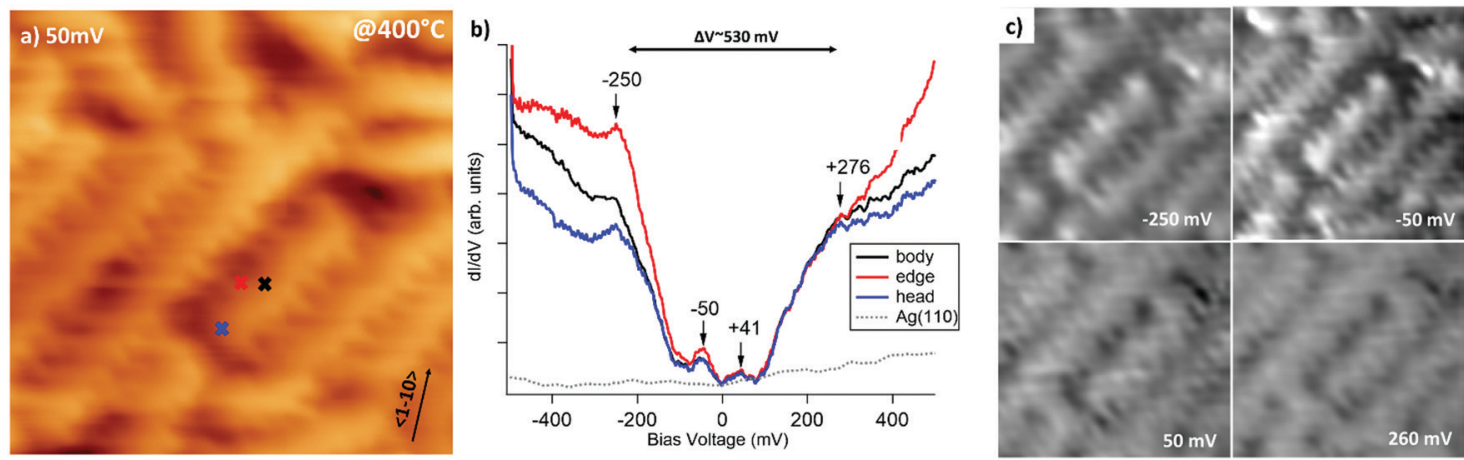

Fig. 4 STS and $\mathrm{d} / / \mathrm{d} V$ map of pristine GNRs in the bias range from $-500 \mathrm{mV}$ to $500 \mathrm{mV}$. (a) Topographical image (image size: $7.0 \times 7.0 \mathrm{~nm}{ }^{2}, V=50 \mathrm{mV}$, $I=0.02 \mathrm{nA}$ ); crosses indicate the sites where point spectra were recorded. (b) Differential conductance spectra recorded at the points marked in (a). The corresponding spectrum recorded on clean $\mathrm{Ag}(110)$ is reported for comparison. A band gap of $\sim 530$ meV was observed throughout the GNR. Localized states at $-50 \mathrm{meV}$ and $+41 \mathrm{meV}$ are present within the gap. Such values were measured reproducibly for $3-5 \mathrm{~nm}$ long GNRs; it is out of the scope of this work to determine a possible length dependence of the energy gap. (c) d//dV maps taken at selected bias voltage suggested by the STS spectra. 
the one hand, the presence of edge states is usually associated to ZGNRs and our nanostructures present a periodic edge site sequence in which two zig-zag sites alternate to one armchair; therefore the almost regular spacing of the brighter spots may correspond to the location of zig-zag sites along the edges and at the heads of the GNR. On the other hand, edge sites should vanish, or at least attenuate, on the axis of the nanoribbon. However, there are no significant differences in the spectra taken at the centre (black trace), at the head (blue trace) and at the border (red trace) of the nanostructure, except that the rise of the LDOS seems to be steeper in the last case. In addition, the bright spots associated to the $-50 \mathrm{meV}$ state in the $\mathrm{d} I / \mathrm{d} V$ maps do not seem to be localized at the edge but rather to be spread across the whole GNR width. Hence, an alternative explanation is that the $-50 \mathrm{meV}$ intensity is related to a localized state due to quantum confinement along the GNR long axis. Though a richer statistics is necessary to confirm this hypothesis, we mention that, in the ultra-narrow limit, hints are present that the gap size can be affected also by the ribbon length. ${ }^{26}$

\section{(iii) Oxygen exposure on GNRs}

When GNRs are grown under UHV conditions, the natural question arises whether their characteristics are modified by the interaction with the atmospheric environment. It is also interesting to understand if small molecules can intercalate below the GNRs as they do for supported graphene monolayers, ${ }^{45,46}$ thus possibly favouring decoupling from the substrate. To give a first answer, we exposed the Ag(110) surface covered by a sub-monolayer of GNRs (Fig. 3a) to $\mathrm{O}_{2}$ at RT
(Fig. 3b) or at $300{ }^{\circ} \mathrm{C}$ (Fig. 3c) in the $10^{-6}$ mbar pressure range. Oxygen was chosen since it is one of the most reactive and abundant gases in air and because it is known to affect the growth of armchair GNRs if present in the UHV chamber above a critical pressure. ${ }^{30}$ The temperature of $300{ }^{\circ} \mathrm{C}$ was chosen in order to favour the mobility of the adsorbate while leaving the structure of the GNRs unaffected (they were produced at $T=400{ }^{\circ} \mathrm{C}$ and prolonged annealing leads only to a reduction in the coverage, without changes in the morphology of the GNRs). Any changes in the surface morphology can therefore be ascribed to the presence of the reactant and not to the effect of prolonged annealing.

The XPS spectra of the $\mathrm{C} 1 \mathrm{~s}$ and $\mathrm{O}$ 1s regions for the GNRs/ $\mathrm{Ag}(110)$ surface before and after $\mathrm{O}_{2}$ exposure are reported in Fig. 5. Since these spectra were recorded with a conventional $\mathrm{X}$-ray source, the resolution is lower than the one of the spectra of Fig. 2. For this reason the $\mathrm{C}-\mathrm{C}$ and $\mathrm{C}-\mathrm{H}$ contribution, differing by only $0.4 \mathrm{eV}$ in binding energy, were not resolved and the major $\mathrm{C}$ 1s signal was fitted as only one component. After synthesis of the GNRs, the $\mathrm{C}$ 1s spectrum is compatible with the one of Fig. 2, while the $\mathrm{O} 1 \mathrm{~s}$ region is completely flat. After exposure to $\mathrm{O}_{2}$ at RT, a minor component at $287.4 \mathrm{eV}$ appears in the $\mathrm{C} 1 \mathrm{~s}$ spectrum while the main C1s line downshifts by $0.25 \mathrm{eV}$. A similar downshift upon oxygen intercalation has already been observed for 2D graphene layers on $\operatorname{Ir}(111)^{47}$ and on polycrystalline $\mathrm{Cu},{ }^{48}$ while a coherent increase in the work function was measured for graphene on $\mathrm{Ru}(0001){ }^{49}$ The effect is interpreted as an indication of a detachment of the graphene layer from the substrate, leading to a lower graphenesubstrate interaction and thus to a reduction of the n-doping
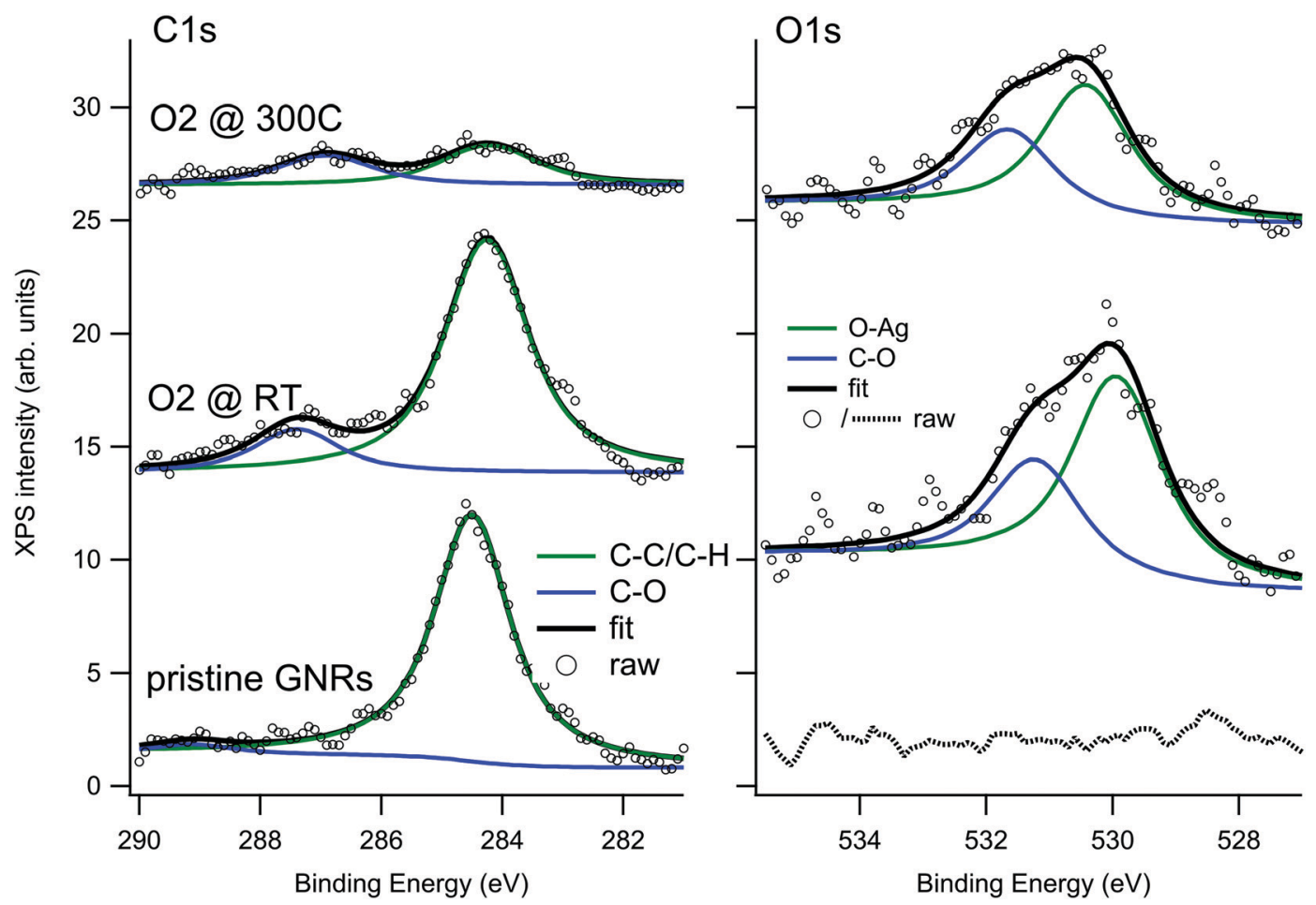

Fig. 5 XPS spectra of the $\mathrm{C}$ 1s (left) and $\mathrm{O}$ 1s (right) regions recorded on the GNRs/Ag(110) surface before and after $\mathrm{O}_{2}$ exposure. 
induced by the presence of the metal substrate. The $\mathrm{O} 1 \mathrm{~s}$ signal can be fitted with two lines at 529.9 and $531.2 \mathrm{eV}$. The former, ascribed to oxygen adatoms on $\mathrm{Ag}$, is expected considering the sticking probability for dissociative adsorption of $\mathrm{O}_{2}$ on $\mathrm{Ag}(110)$ at RT $(0.1 \%),{ }^{50}$ the presence of bare areas of the sample and the large oxygen exposure we performed. The features at 287.4 and $531.2 \mathrm{eV}$, on the contrary, are compatible with the formation of $\mathrm{C}-\mathrm{O}$ bonds ${ }^{51-54}$ and suggest that some interaction of dissociated oxygen atoms with the GNRs occurs. Reaction of oxygen atoms with pyrene compounds, especially in presence of a metal substrate, is possible and may lead to different oxygenated pyrene species, such as pyrene containing hydroxyl, carbonyl or epoxy functionalities. From the observed binding energies, the formation of carbonyl groups is the most probable. Additional $\mathrm{O}_{2}$ exposure at $300{ }^{\circ} \mathrm{C}$ causes a reduction of the C1s signal, indicating partial desorption of the nanostructures, and a relative increase of the high $E_{\mathrm{b}}$ components both in the $\mathrm{C}$ 1s and $\mathrm{O}$ 1s regions.

The corresponding STM experiment is reported in the overviews of Fig. 3 and in the enlargements of Fig. 6. By comparing the morphology of the GNRs/Ag(110) surface upon the different treatments, we note that, apparently, oxygen exposure at RT has the main effect of inducing some disorder in the nanostructure arrays, which now show little preferential orientation on the

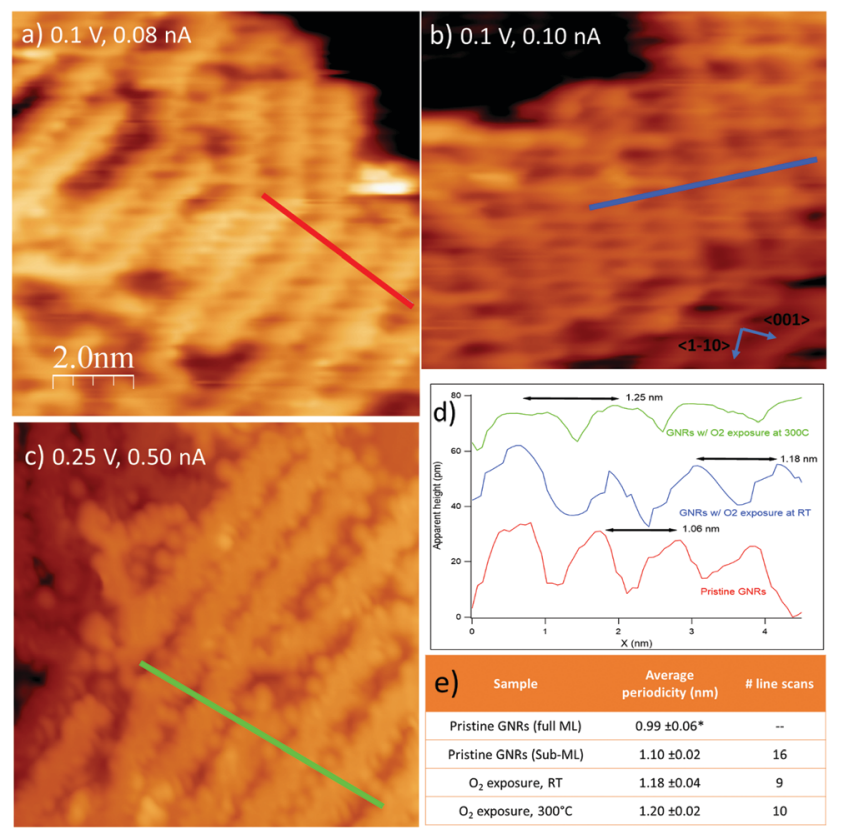

Fig. 6 Close up images of the three preparations in Fig. 3 with corresponding line scans indicating the periodicity of the GNRs. (A) Pristine GNRs; (b) same as (a) after exposure to $\mathrm{O}_{2}$ at RT; (c) same as (a) after exposure to $\mathrm{O}_{2}$ at $300{ }^{\circ} \mathrm{C}$. In this preparation the small features in-between the GNRs are evident. (d) Line scans cut along the paths marked in panels $(a-c)$ showing that the separation between adjacent GNRs increases after $\mathrm{O}_{2}$ exposure. (e) Table summarizing the average separation between GNRs in ordered arrays, together with the number of line scans considered for each preparation (corresponding to the number of GNRs arrays considered). Values are given with their standard error. Data indicated with * are taken from ref. 12 surface. There are no additional features that can be related to oxygen adsorption but the lower stability of the tip and the worse quality of the images (see also enlarged image in Fig. 6b) witness that oxygen must be present on the surface, coherently with the XPS information of Fig. 5. On the contrary, peculiar morphological changes are observed after dosing oxygen at $300{ }^{\circ} \mathrm{C}$. As evident in the STM image of Fig. $3 \mathrm{c}$ and in the enlargement reported in Fig. 6c, besides the GNRs, several small round features (indicated by the arrows in Fig. 3c) are now present at the surface, either in-between adjacent nanostructures or on bare $\mathrm{Ag}$ areas, where they organize in short rows. We note that apparently similar features in-between the GNRs were also observed for the full monolayer of pristine GNRs but not for the sub-monolayer preparation of Fig. 3a. For the full monolayer coverage, they were identified with residual $\mathrm{Br}$ and $\mathrm{Ag}$ adatoms deriving from the thermal dissolution of the organometallic chains: ${ }^{12}$ the $\mathrm{Ag}$ adatoms failed to migrate back to the step edges of the $\mathrm{Ag}(110)$ planes due to the compact arrangement of the GNRs. This constrain is apparently removed for the less dense sub-monolayer of Fig. 3a. Upon oxygen exposure at $300{ }^{\circ} \mathrm{C}$, the small bright dots are more abundant than they were on the full monolayer of pristine GNRs. ${ }^{12}$ They are randomly distributed along the edges of the nanostructures but not necessarily close to them. Therefore, it seems reasonable to identify such bright dots with Agcontaining features stabilized at the surface by the presence of oxygen. Indeed $\mathrm{Ag}$ is prone to react with oxygen to form a wide zoology of Ag-O complexes. ${ }^{31,32}$ The height profile of the isolated dots (not shown) protrudes by $\sim 0.4 \AA$ from the bare $\mathrm{Ag}$ plane; it has therefore a shape similar to the one of $\mathrm{O}-\mathrm{Ag}-\mathrm{O}$ added rows and suggests that the observed protrusions consist of $\mathrm{Ag}$ and $\mathrm{O}$ adatoms.

Finally, we considered the small arrays of parallel GNRs, which are present for all the three preparations reported in Fig. 6 and we measured the separation between adjacent GNRs. Sample line scans drawn along the paths marked in panels $(\mathrm{a}-\mathrm{c})$ are reported in panel $(\mathrm{d})$, while the table in panel (e) summarizes the outcome of a statistical analysis over several ordered arrays. The corresponding value measured for full monolayer coverage is also reported for comparison. ${ }^{12}$ Firstly, we note that, also for the sub-monolayer, GNRs tend to form arrays, though less densely packed than for the full monolayer coverage. ${ }^{12}$ The distance between adjacent nanostructures further increases upon oxygen exposure: the line scans present a less regular profile (the distance between maxima is not always the same, in accord with the more disordered distribution of the nanostructures) and, on average, the spacing between adjacent structures increases by $\sim 10 \%$ with respect to the pristine sample. We remark that this effect must be certainly ascribed to oxygen exposure; in fact, the samples in Fig. $3 \mathrm{~b}, \mathrm{c}$ and $6 \mathrm{~b}$, c were obtained by exposing the pristine sample of Fig. 3a and 6a, so that the initial density of GNRs is exactly the same. The larger separation between adjacent nanoribbons upon oxygen adsorption suggests that the $\mathrm{O}$ adatoms adsorbed on the first $\mathrm{Ag}$ layer or the $\mathrm{O}-\mathrm{Ag}-\mathrm{O}$ complexes act as "spacers". It also indicates that, following oxygen 
exposure, the bonding of the nanostructure to the substrate gets weaker and the GNRs can displace along the surface without changing their geometry. This observation perfectly matches with the downshift of the main C 1s line observed in the corresponding XPS experiment (Fig. 5). The overall picture suggests therefore that the GNR-Ag interaction is weakened upon $\mathrm{O}_{2}$ exposure; in view of the observed $\mathrm{C}$ 1s core level shift, this effect is due more likely to oxygen intercalation than to modifications of the metal surface upon oxygen adsorption.

STS spectra would be extremely useful to determine if this weakening affects the width of the band-gap or its position with respect to the Fermi edge but, unfortunately, we were unable to record them with a useful signal-to-noise ratio due to the instability of the tip for samples exposed to oxygen. On the contrary, $\mathrm{d} I / \mathrm{d} V$ maps could be acquired in the bias range $-1.0 \mathrm{~V}$ $<V<+1.0 \mathrm{~V}$ after dosing $\mathrm{O}_{2}$ at $300{ }^{\circ} \mathrm{C}$.

Fig. 7 shows a comparison between such maps recorded near the band-gap edges for the pristine GNRs sample and for the same sample after exposure to $\mathrm{O}_{2}$ at $300{ }^{\circ} \mathrm{C}$. We first observe a contrast change between positive (empty states) and negative (filled states) bias for the pristine GNRs. Indeed, at $-250 \mathrm{mV}$ the nanostructures appear narrower and the measured corrugation is larger than at $+250 \mathrm{meV}$. This effect is much reduced after exposure to $\mathrm{O}_{2}$; in this case the images appear with a very similar contrast and the small differences at the edges are a consequence of the derivative operation. In addition, the nanostructures appear wider and their edges are much better defined than on the pristine surface. The effect goes well beyond the improved quality of the tip in this image. We recall
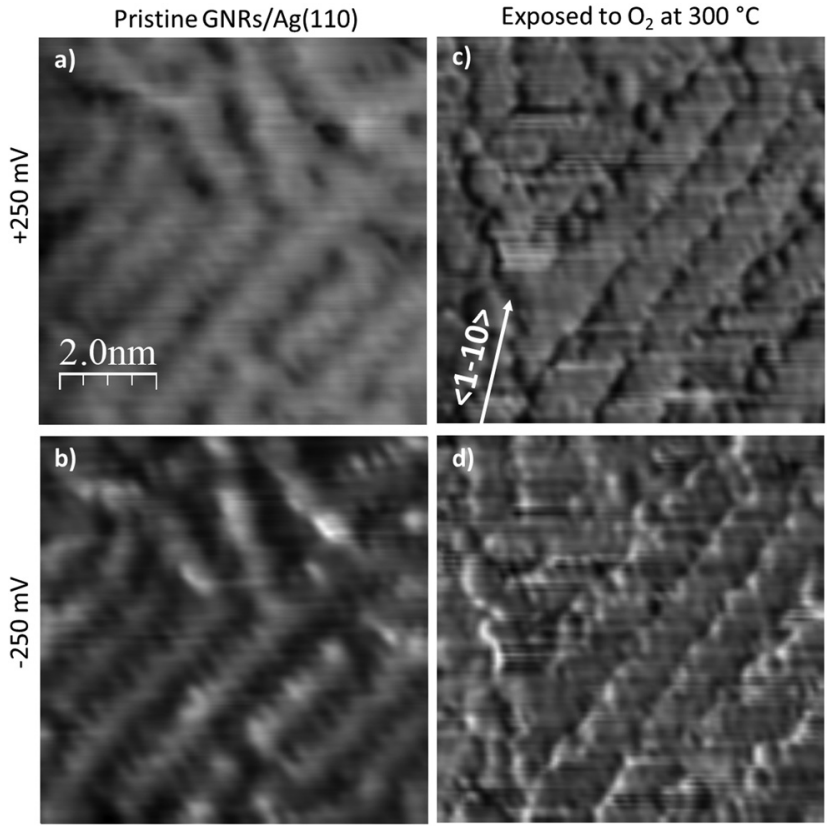

Fig. 7 Comparison of the $\mathrm{d} / / \mathrm{d} V$ maps of GNRs pristine and after exposure to oxygen at $300{ }^{\circ} \mathrm{C}$. Bias voltages corresponding to the edges of the energy gap measured for the pristine sample are presented. The corresponding topographical images are those of Fig. $4 a$ and $5 \mathrm{c}$. Z-scale is the same for all images. that oxygen has intercalated under the GNRs and that some $\mathrm{O}$ adatoms must have reacted with the nanostructures, as witnessed by the presence of $\mathrm{C} 1 \mathrm{~s}$ and $\mathrm{O} 1 \mathrm{~s}$ components compatible with the formation of carbonyl groups in the XPS spectra. It is possible that these phenomena cause a better confinement of electrons within the GNR.

Finally, we mention that previous literature on AGNRs reports that the nanostructures are affected by the presence of gases (water or oxygen) during the surface assisted synthesis $^{30}$ but that, if grown under clean UHV conditions, their properties are stable even at atmospheric pressure. ${ }^{29}$ Our results are not in contradiction with these works since they refer to the morphology of the GNRs, which is also preserved in our case, while they did not perform any XPS analysis on the investigated systems.

\section{Conclusion}

Mixed edge-site, $1.0 \mathrm{~nm}$ wide GNRs grown on Ag(110) were characterized by LT-STM, STS and XPS and the effect of oxygen exposure in the $10^{-6}$ mbar range was addressed. An energy gap of $\sim 530 \mathrm{meV}$ is measured for the pristine GNRs, in line with the values reported in literature, and two localized states, most probably related to quantum confinement, are found inside the gap.

Upon oxygen exposure, the surface appears more disordered and the average separation between adjacent GNRs increases by $\sim 10 \%$, while a downshift of the main $\mathrm{C} 1 \mathrm{~s}$ peak is observed in the XPS spectrum. When $\mathrm{O}_{2}$ is dosed on the sample at $T=300{ }^{\circ} \mathrm{C}$, a significant modification of the surface occurs, since Ag-O complexes appear on the surface both in-between the nanoribbons and at bare Ag patches. The overall appearance of the GNRs in morphological images does not change significantly, but the formation of carboxyl groups is detected by photoemission spectroscopy, suggesting that some $\mathrm{C}$ atoms of the GNRs have reacted with oxygen adatoms. Coherently, $\mathrm{d} I / \mathrm{d} V$ maps recorded on the pristine and oxygen-exposed samples are different, suggesting that oxygen must play an active role. Its adsorption reduces the interaction between the nanostructures and the $\operatorname{Ag}(110)$ substrate and thus favours their displacement and electronic decoupling.

These results clearly demonstrate that care must be taken about the modification of supported GNRs upon exposure to reactive gases, not only for their possible interaction with the nanostructures, but also for the changes induced by the reactant on the whole system. This insight opens important perspectives for the optimization of the transfer procedure of the nanoribbons on a different substrate, as well as for operating nanostructure-based devices under realistic conditions.

\section{Conflicts of interest}

There are no conflicts to declare. 


\section{Acknowledgements}

We thank Angelique J. Lusuan and Jade Chalancon for taking part to the initial stages of the experiment and to acquisition of STS spectra, respectively. We acknowledge financial support from MIUR through projects PRIN2017 project no. 2017NYPHN8 and FIRB Futuro in Ricerca 2012 No. RBFR128BEC. L. S., M. S., L. V. and M. R. acknowledge funding from Fondazione Compagnia di S. Paolo through project MC-nano. I. P., E. M., S. N. and F. B. acknowledge funding from EUROFEL project (RoadMap Esfri). We acknowledge Elettra Sincrotrone Trieste for providing access to its synchrotron radiation facilities. P. W. acknowledges financial support from the European Social Fund through the Operational Programme Knowledge Education Development project "Interdisciplinary $\mathrm{PhD}$ studies in nanotechnology" (project no. POWR.03.02.00-00-I032/16) and, partially, from the National Science Centre of Poland through the OPUS project no. 2014/15/B/ST3/02927.

\section{References}

1 X. Li, X. Wang, L. Zhang, S. Lee and H. Dai, Chemically derived, ultrasmooth graphene nanoribbon semiconductors, Science, 2008, 319, 1229-1232.

2 K. Nakada, M. Fujita, G. Dresselhaus and M. S. Dresselhaus, Edge state in graphene ribbons: nanometer size effect and edge shape dependence, Phys. Rev. B: Condens. Matter Mater. Phys., 1996, 54, 17954-17961.

3 Y.-C. Chen, D. G. de Oteyza, Z. Pedramrazi, C. Chen, F. R. Fischer and M. F. Crommie, Tuning the band gap of graphene nanoribbons synthesized from molecular precursors, ACS Nano, 2013, 7, 6123-6128.

4 X. Wang, Y. Ouyang, X. Li, H. Wang, J. Guo and H. Dai, Room-temperature all-semiconducting sub-10-nm graphene nanoribbon field-effect transistors, Phys. Rev. Lett., 2008, 100, 206803.

5 J. Baringhaus, M. Ruan, F. Edler, A. Tejeda, M. Sicot, A. Taleb-Ibrahimi, A.-P. Li, Z. Jiang, E. H. Conrad, C. Berger, C. Tegenkamp and W. A. de Heer, Exceptional ballistic transport in epitaxial graphene nanoribbons, Nature, 2014, 506, 349-354.

6 M. Y. Han, B. Özyilmaz, Y. Zhang and P. Kim, Energy bandgap engineering of graphene nanoribbons, Phys. Rev. Lett., 2007, 98, 206805.

7 L. Jiao, L. Zhang, X. Wang, G. Diankov and H. Dai, Narrow graphene nanoribbons from carbon nanotubes, Nature, 2009, 458, 877-880.

8 D. V. Kosynkin, A. L. Higginbotham, A. Sinitskii, J. R. Lomeda, A. Dimiev, B. K. Price and J. M. Tour, Longitudinal unzipping of carbon nanotubes to form graphene nanoribbons, Nature, 2009, 458, 872-876.

9 X. Yang, X. Dou, A. Rouhanipour, L. Zhi, H. J. Räder and K. Müllen, Two-dimensional graphene nanoribbons, J. Am. Chem. Soc., 2008, 130, 4216-4217.

10 P. Han, K. Akagi, F. Federici Canova, H. Mutoh, S. Shiraki, K. Iwaya, P. S. Weiss, N. Asao and T. Hitosugi, Bottom-up graphene-nanoribbon fabrication reveals chiral edges and enantioselectivity, ACS Nano, 2014, 8, 9181-9187.

11 J. Cai, P. Ruffieux, R. Jaafar, M. Bieri, T. Braun, S. Blankenburg, M. Muoth, A. P. Seitsonen, M. Saleh, X. Feng, K. Müllen and R. Fasel, Atomically precise bottom-up fabrication of graphene nanoribbons, Nature, 2010, 466, 470-473.

12 M. Smerieri, I. Pis, L. Ferrighi, S. Nappini, A. J. Lusuan, C. di Valentin, L. Vaghi, A. Papagni, M. Cattelan, S. Agnoli, E. Magnano, F. Bondino and L. Savio, Synthesis of graphene nanoribbons with a defined mixed edge-site sequence by surface assisted polymerization of (1,6)-dibromopyrene on $\mathrm{Ag}(110)$, Nanoscale, 2016, 8, 17843-17853.

13 G. Franc and A. Gourdon, Covalent networks through onsurface chemistry in ultra-high vacuum: State-of-the-art and recent developments, Phys. Chem. Chem. Phys., 2011, 13, 14283-14292.

14 S.-W. Hla, L. Bartels, G. Meyer and K.-H. Rieder, Inducing all steps of a chemical reaction with the scanning tunneling microscope tip: towards single molecule engineering, Phys. Rev. Lett., 2000, 85, 2777-2780.

15 A. Lafferentz, M. Wenderoth, S. Lounis, P. Zahn, N. Quaas, R. G. Ulbrich, P. H. Dederichs and S. Blugel, Conductance of a single conjugated polymer as a continuous function of its length, Science, 2009, 323, 1190-1193.

$16 \mathrm{X}$. Zhou and G. Yu, Modified engineering of graphene nanoribbons prepared via on-surface synthesis, Adv. Mater., 2020, 32, 1-24.

17 J. Liu, B. W. Li, Y. Z. Tan, A. Giannakopoulos, C. SanchezSanchez, D. Beljonne, P. Ruffieux, R. Fasel, X. Feng and K. Müllen, Toward cove-edged low band gap graphene nanoribbons, J. Am. Chem. Soc., 2015, 137, 6097-6103.

18 Q. Fan, J. M. Gottfried and J. Zhu, Surface-catalyzed C-C covalent coupling strategies toward the synthesis of lowdimensional carbon-based nanostructures, Acc. Chem. Res., 2015, 48, 2484-2494.

19 P. Ruffieux, S. Wang, B. Yang, C. Sanchez-Sanchez, J. Liu, T. Dienel, L. Talirz, P. Shinde, C. A. Pignedoli, D. Passerone, T. Dumslaff, X. Feng, K. Müllen and R. Fasel, On-surface synthesis of graphene nanoribbons with zigzag edge topology, Nature, 2016, 531, 489-492.

20 K. A. Simonov, N. A. Vinogradov, A. S. Vinogradov, A. V. Generalov, E. M. Zagrebina, N. Mårtensson, A. A. Cafolla, T. Carpy, J. P. Cunniffe and A. B. Preobrajenski, Effect of substrate chemistry on the bottom-up fabrication of graphene nanoribbons: combined core-level spectroscopy and STM study, J. Phys. Chem. C, 2014, 118, 12532-12540.

21 K. A. Simonov, N. A. Vinogradov, A. S. Vinogradov, A. V. Generalov, E. M. Zagrebina, G. I. Svirskiy, A. A. Cafolla, T. Carpy, J. P. Cunniffe, T. Taketsugu, A. Lyalin, N. Mårtensson and A. B. Preobrajenski, From graphene nanoribbons on $\mathrm{Cu}(111)$ to nanographene on $\mathrm{Cu}(110)$ : critical role of substrate structure in the bottom-up fabrication strategy, ACS Nano, 2015, 9, 8997-9011.

22 Y. Li, W. Zhang, M. Morgenstern and R. Mazzarello, Electronic and magnetic properties of zigzag graphene 
nanoribbons on the (111) surface of $\mathrm{Cu}, \mathrm{Ag}$, and $\mathrm{Au}$, Phys. Rev. Lett., 2013, 110, 1-5.

23 L. Yan and P. Liljeroth, Engineered electronic states in atomically precise artificial lattices and graphene nanoribbons, Adv. Phys. X, 2019, 4, 1651672.

24 M. Smerieri, I. Píš, L. Ferrighi, S. Nappini, A. Lusuan, L. Vattuone, L. Vaghi, A. Papagni, E. Magnano, C. Di Valentin, F. Bondino and L. Savio, Synthesis of corrugated C-based nanostructures by Br-corannulene oligomerization, Phys. Chem. Chem. Phys., 2018, 20, 26161-26172.

25 I. Píš, L. Ferrighi, T. H. Nguyen, S. Nappini, L. Vaghi, A. Basagni, E. Magnano, A. Papagni, F. Sedona, C. Di Valentin, S. Agnoli and F. Bondino, Surface-confined polymerization of halogenated polyacenes: the case of dibromotetracene on $\mathrm{Ag}(110)$, J. Phys. Chem. C, 2016, 120, 4909-4918.

26 A. Kimouche, M. M. Ervasti, R. Drost, S. Halonen, A. Harju, P. M. Joensuu, J. Sainio and P. Liljeroth, Ultra-narrow metallic armchair graphene nanoribbons, Nat. Commun., 2015, 6, 10177.

27 J. Cai, C. A. Pignedoli, L. Talirz, P. Ruffieux, H. Söde, L. Liang, V. Meunier, R. Berger, R. Li, X. Feng, K. Müllen and R. Fasel, Graphene nanoribbon heterojunctions, Nat. Nanotechnol., 2014, 9, 896-900.

28 S. Kawai, S. Saito, S. Osumi, S. Yamaguchi, A. S. Foster, P. Spijker and E. Meyer, Atomically controlled substitutional boron-doping of graphene nanoribbons, Nat. Commun., 2015, 6, 8098.

29 G. Borin Barin, A. Fairbrother, L. Rotach, M. Bayle, M. Paillet, L. Liang, V. Meunier, R. Hauert, T. Dumslaff, A. Narita, K. Müllen, H. Sahabudeen, R. Berger, X. Feng, R. Fasel and P. Ruffieux, Surface-synthesized graphene nanoribbons for room temperature switching devices: substrate transfer and ex situ characterization, ACS Appl. Nano Mater., 2019, 2, 2184-2192.

30 A. Fairbrother, J. R. Sanchez-Valencia, B. Lauber, I. Shorubalko, P. Ruffieux, T. Hintermann and R. Fasel, High vacuum synthesis and ambient stability of bottom-up graphene nanoribbons, Nanoscale, 2017, 9, 2785-2792.

31 T. B. Rawal, M. Smerieri, J. Pal, S. Hong, M. Alatalo, L. Savio, L. Vattuone, T. S. Rahman and M. Rocca, Deciphering complex features in STM images of O adatoms on $\mathrm{Ag}(110)$, Phys. Rev. B, 2018, 98, 35405.

32 L. Savio, M. Smerieri, L. Vattuone, A. Gussoni, C. Tassistro and M. Rocca, STM study of hydroxyl formation at $\mathrm{O} / \mathrm{Ag}(110)$, Phys. Rev. B: Condens. Matter Mater. Phys., 2006, 74, 1-6.

33 I. Horcas, R. Fernandez, J. Gomez-Rodriguez, J. Colchero, J. Gomez-Herrero and A. Baro, Rev. Sci. Instrum., 2007, 78, 013705.

34 S. Doniach and M. Sunjic, Many-electron singularity in X-ray photoemission and X-ray line spectra from metals, J. Phys. C: Solid State Phys., 1970, 3, 285-291.

35 J. J. Joyce, M. Del Giudice and J. H. Weaver, Quantitative analysis of synchrotron radiation photoemission core level data, J. Electron Spectrosc. Relat. Phenom., 1989, 49, 31-45.

36 F. C. Henn, M. E. Bussell and C. T. Campbell, A simple means for reproducibly dosing low vapor pressure and/or reactive gases to surfaces in ultrahigh vacuum, J. Vac. Sci. Technol., A, 1991, 9, 10-13.

37 C. Moreno, M. Panighel, M. Vilas-Varela, G. Sauthier, M. Tenorio, G. Ceballos, D. Peña and A. Mugarza, Critical role of phenyl substitution and catalytic substrate in the surface-assisted polymerization of dibromobianthracene derivatives, Chem. Mater., 2019, 31, 331-341.

38 J. Hu, J. Hu, Z. Zhang, K. Shen, Z. Liang, H. Zhang, Q. Tian, P. Wang, Z. Jiang, H. Huang, J. W. Wells and F. Song, Ullmann coupling of 2,7-dibromopyrene on $\mathrm{Au}(111)$ assisted by surface adatoms, Appl. Surf. Sci., 2020, 513, 145797.

39 A. Basagni, F. Sedona, C. A. Pignedoli, M. Cattelan, L. Nicolas, M. Casarin and M. Sambi, Molecules-oligomers-nanowires-graphene nanoribbons: a bottom-up stepwise on-surface covalent synthesis preserving long-range order, J. Am. Chem. Soc., 2015, 137, 1802-1808.

40 A. Mairena, M. Baljozovic, M. Kawecki, K. Grenader, M. Wienke, K. Martin, L. Bernard, N. Avarvari, A. Terfort, K.-H. Ernst and C. Wäckerlin, The fate of bromine after temperature-induced dehydrogenation of on-surface synthesized bisheptahelicene, Chem. Sci., 2019, 10, 2998-3004.

41 G. Giovannetti, P. A. Khomyakov, G. Brocks, V. M. Karpan, J. Van Den Brink and P. J. Kelly, Doping graphene with metal contacts, Phys. Rev. Lett., 2008, 101, 3-6.

42 A. Dahal and M. Batzill, Graphene-nickel interfaces: a review, Nanoscale, 2014, 6, 2548-2562.

43 L. G. Bulusheva, V. E. Arkhipov, K. M. Popov, V. I. Sysoev, A. A. Makarova and A. V. Okotrub, Electronic structure of nitrogen-and phosphorus-doped graphenes grown by chemical vapor deposition method, Materials, 2020, 13, 1173.

44 R. Wu, J. Ren, L. Dong, Y. Wang, Q. Huan and H. J. Gao, Quasi-free-standing graphene nano-islands on $\mathrm{Ag}(110)$, grown from solid carbon source, Appl. Phys. Lett., 2017, 110, 213107.

45 C. Riedl, C. Coletti, T. Iwasaki, A. A. Zakharov and U. Starke, Quasi-free-standing epitaxial graphene on SiC obtained by hydrogen intercalation, Phys. Rev. Lett., 2009, 103, 2-5.

46 S. L. Kovalenko, B. V. Andryushechkin and K. N. Eltsov, STM study of oxygen intercalation at the graphene/Ni(111) interface, Carbon, 2020, 164, 198-206.

47 R. Larciprete, S. Ulstrup, P. Lacovig, M. Dalmiglio, M. Bianchi, F. Mazzola, L. Hornekær, F. Orlando, A. Baraldi, P. Hofmann and S. Lizzit, Oxygen switching of the epitaxial graphene-metal interaction, ACS Nano, 2012, 6, 9551-9558.

48 R. Blume, P. R. Kidambi, B. C. Bayer, R. S. Weatherup, Z. J. Wang, G. Weinberg, M. G. Willinger, M. Greiner, S. Hofmann, A. Knop-Gericke and R. Schlögl, The influence of intercalated oxygen on the properties of graphene on polycrystalline $\mathrm{Cu}$ under various environmental conditions, Phys. Chem. Chem. Phys., 2014, 16, 25989-26003.

49 P. Sutter, P. Albrecht, X. Tong and E. Sutter, Mechanical decoupling of graphene from $\mathrm{Ru}(0001)$ by interfacial 
reaction with oxygen, J. Phys. Chem. C, 2013, 117, 6320-6324.

50 L. Vattuone, M. Rocca, C. Boragno and U. Valbusa, Initial sticking coefficient of $\mathrm{O}_{2}$ on $\mathrm{Ag}(110)$, J. Chem. Phys., 1994, 101, 713-725.

51 Y. C. G. Kwan, G. M. Ng and C. H. A. Huan, Identification of functional groups and determination of carboxyl formation temperature in graphene oxide using the XPS O 1s spectrum, Thin Solid Films, 2015, 590, 40-48.

52 A. Ganguly, S. Sharma, P. Papakonstantinou and J. Hamilton, Probing the thermal deoxygenation of graphene oxide using high-resolution in situ X-ray-based spectroscopies, J. Phys. Chem. C, 2011, 115, 17009-17019.

53 U. Zielke, K. J. Hüttinger and W. P. Hoffman, Surfaceoxidized carbon fibers: I. Surface structure and chemistry, Carbon, 1996, 34, 983-998.

54 J. Matthew, Surface analysis by Auger and X-ray photoelectron spectroscopy. D. Briggs and J. T. Grant (eds). IMPublications, Chichester, UK and SurfaceSpectra, Manchester, UK, 2003. 900 pp., ISBN 1-901019-04-7, 900 pp, Surf. Interface Anal., 2004, 36, 1647. 